institute, as part of multicentre studies performed between January 2002 and December 2009. Rest and vasodilator SPECT was performed after injection of $99 \mathrm{mTc}$-sestamibi using the standard technique on separate days. Coronary Angiography (CA) was performed within thirty days of stress imaging. We calculated the ratio of the number of abnormal segments (at rest and/or stress) to the total number of segments expressed as $\%$ for both MCE and SPECT in order to obtain a uniform assessment of the total ischaemic and scar burden (MCE and SPECT indexes). This population was followed up in 2016 to obtain a long term prognostic value of MCE and SPECT for hard events, all-cause mortality and non-fatal myocardial infarction (NFMI).

Results Of the 277 patients who were analysed, 262 followed up and 15 were lost to follow up (5.4\%). The mean age was 63.4 years and $186(71.8 \%)$ patients were male, $82(31.7 \%)$ had diabetes, 180 (69.5\%) hypertension, 189 (73\%) dyslipidaemia, $26(10 \%)$ family history of CAD (FHCAD) and 64 (24.7\%) were smokers. Prior CAD (angina, known acute myocardial infarction (AMI) or coronary revascularisation) was present in $178(68.7 \%)$ patients, left ventricular systolic dysfunction in $32(12.4 \%)$ and chronic kidney disease in 16 (6.2\%).

Over a mean follow-up period of 80 months(6.6 years) \pm 6 months, 18 patients suffered NFMI and 29 died $(18 \%$ hard events, annualised hard events 2.7\%).

Both MCE and SPECT indexes were significant predictors on univariate analysis for all-cause mortality $(p=0.008$ and $\mathrm{p}=0.035$ respectively), but MCE index was the only independent predictor for hard events (HR 3.711, 95\% CI(1.1312.14), $\mathrm{p}=0.03)$. Figure 1 demonstrates the Kaplan-Meier curve for the long-term prognostic value in all-cause mortality and NFMI of abnormal versus normal MCE. The annualised event rate for the abnormal MCE is $3.8 \%$ versus $1.0 \%$ for the normal MCE.

Conclusion This is the first study to our knowledge that investigated the long-term prognostic value of SPECT and MCE in patients with suspected or known CAD. MCE was the only independent predictor of hard events. These results further support the routine use of MCE and not SPECT for the long-term prognostication of patients with known or suspected CAD.

\section{CLINICAL OUTCOME AND COST-EFFECTIVENESS OF PERFORMING CARDIAC INVESTIGATIONS IN A VERY LOW LIKEHOOD OF CORONARY ARTERY DISEASE POPULATION ACCORDING TO NICE AND ESC RISK PREDICTION MODELS}

Nikos Karogiannis*, Konstantinos Zacharias, Anastasia Vamvakidou, Sothinathan Gurunathan, Roxy Senior. Northwick Park Hospital

10.1136/heartjnl-2017-311726.107

Background The NICE (National Institute for Health and Care Excellence) guidance for the management of recent-onset chest pain in 2010, recommended no routine cardiac investigations for patients with risk of coronary artery disease (CAD) less than 10\%. The ESC (European Society of Cardiology) guidelines for stable angina in 2013, proposed no further testing in patients with a pretest probability below $15 \%$. The management of patients with very low risk for CAD can be challenging, particularly when there is high clinical suspicion, family history of premature CAD (FHCAD) or ethnicity background with high prevalence of CAD. We sought to evaluate the clinical and economic impact of performing tests to diagnose CAD in this population.

Methods We retrospectively analysed patients with very low risk for $\mathrm{CAD}$ who attended rapid access chest pain clinic (RACPC) due to recent onset chest pain. The likelihood of CAD was estimated by the NICE modified Diamond-Forrester formula and the ESC pretest probability formula and it was below $10 \%$ and $15 \%$ respectively. According to the guidelines, no further investigations are recommended for this group. Patients underwent exercise ECG (ExECG) or/and stress echocardiography (SE) mainly due to increased clinical suspicion. Coronary Angiography (CA) was subsequently performed, if it was clinically indicated. This population was followed-up for all-cause mortality, myocardial infarction (MI) and revascularisation. Cost-analysis was also performed.

Results Over a period of 12 months, 279 patients underwent ExECG $[171(61,3 \%)$ negative, $13(4,6 \%)$ positive and 56 $(20 \%)$ inconclusive] or/and SE [72 (25,8\%) negative and 5 $(1.7 \%)$ positive]. Coronary angiography performed in 26 patients $(9.3 \%)$ and significant CAD was identified in one patient (0.3\%). Regarding risk factors, 88 (31.5\%) patients had FHCAD, 66 (23.6\%) hypertension, 30 (10.7\%) dyslipidaemia, $17(6.1 \%)$ diabetes and $10(3.5 \%)$ were smokers. The ethnicity distribution predominantly consisted of South Asians (49.8\%).

Over 55 months (4.5 years) \pm 5 months of follow up, two patients were diagnosed with myocardial infarction $(0.7 \%)$ but only one had significant $\mathrm{CAD}$ and subsequent revascularisation $(0.3 \%)$. Both patients had ExECG initially which was negative. Also, two patients died $(0.7 \%)$ both from non-cardiovascular cause.

A total cost of $£ 69,060$ was spent in these tests with a mean cost of $£ 247$ per patient.

Conclusion The above findings support the rationale of avoiding further cardiac investigations in patients who are at very low risk of CAD as it is recommended by NICE and ESC guidelines. The cost-effectiveness of this strategy is welldepicted in previous studies and our findings are consistent with these results. Current guidance for the management of these patients seems that it is reassuringly applicable in ethnicities with high prevalence of CAD.

\section{THE PROGNOSTIC VALUE OF STRESS CMR IN A TERTIARY CENTRE}

Mohammed Meah* ${ }^{*}$ Wern Ding, Jonathan Hasleton, James McShane, Joseph Mills. NHS

\subsection{6/heartjnl-2017-311726.108}

Background Stress cardiac magnetic resonance imaging (CMR) is increasingly being used in the assessment of myocardial perfusion at rest and in response to exertion. It provides information on the presence or absence of functionally significant coronary artery disease and allows us to predict the benefit of revascularisation. The aim of this study is to assess the accuracy and prognostic value of stress CMR in our tertiary centre.

Method Retrospective single centre study of every adenosine stress CMR done for patients suspected of having ischaemic heart disease between January 2012 and December 2014 $(n=525)$. Reports were reviewed and patients categorised into 
"reversible ischaemia" (RI, n=94), "non-reversible ischaemia" (NRI, $n=146)$, and "no ischaemia" (NoI, n=285). Follow up data was collected on all-cause-mortality, hospital admissions with acute coronary syndromes and admissions for revascularisation. A Kaplan-Meier survival analysis was done using the composite end point of admission with acute coronary syndrome or for revascularisation.

Using angiography as the reference standard in those that had concurrent angiograms $(n=83)$ sensitivity and specificity was calculated. Where pressure wire studies (PWS) were undertaken $(n=15)$ results were correlated with CMR reports. Results 525 patients were included in the final analysis. Median follow-up of 15 months. A total of $46 \%$ had a perfusion defect on CMR. During the follow up, the distribution in composite outcomes in those with and those without defect was highly significant $(p<0.0001)$ (see figure 1 and table 1$)$.

83 patients had concurrent angiograms, CMR demonstrated 91.1\% sensitivity, $77.8 \%$ specificity, $89.5 \%$ positive predictive value and $80.8 \%$ negative predictive value (see table 2 ). There was $100 \%$ correlation between CMR and PWS in those reported as having RI $(n=5)$ and $80 \%$ correlation in those who had NoI $(n=5)$. All cause mortality in the NoI group was $2.97 \%(8 / 269)$, and in the ischaemia groups was $4.66 \%$ (11/236), though there was no statistical significance our study was limited by the small number of deaths.

Conclusion There is good correlation between pressure wire studies and stress CMR, but our sample size is small, larger studies (such as MR-INFORM) are needed to accurately inform the role of CMR perfusion in investigating and managing coronary artery disease. Patients with a normal stress CMR scan appear to have excellent prognosis.

\begin{tabular}{ll} 
Abstract 109 Table 1 & \\
\hline RI vs Nol & $11.1(5.8-21.9)$ \\
\hline NRI vs Nol & $3.3(1.9-5.7)$ \\
\hline
\end{tabular}

\begin{tabular}{lll} 
Abstract 109 Table 2 & & \\
\hline CMR positive (n) & 51 & 6 \\
\hline CMR negative (n) & 5 & 21 \\
\hline
\end{tabular}

\section{LATE GADOLINIUM ENHANCEMENT IN PRIMARY DEGENERATIVE MITRAL REGURGITATION PREDICTS ADVERSE RIGHT VENTRICULAR REMODELLING AND EXERCISE INDUCED PULMONARY HYPERTENSION}

${ }^{1}$ Boyang Liu*, ${ }^{2}$ Nicola Edwards, ${ }^{2}$ Moninder Bhabra, ${ }^{3}$ Thomas Barker, ${ }^{3}$ Ramesh Patel, ${ }^{2}$ Richard Steeds. ${ }^{1}$ University of Birmingham; ${ }^{2}$ University Hospital Birmingham; ${ }^{3}$ University Hospital Coventry

\subsection{6/heartjnl-2017-311726.109}

Introduction The optimal timing of surgery in asymptomatic severe primary mitral regurgitation (MR) continues to be challenging. Late gadolinium enhancement (LGE) on cardiac magnetic resonance imaging (CMR) is an established imaging biomarker of irreversible myocardial fibrosis within the ventricles that is associated with worse prognosis in a broad range of cardiovascular diseases. There are limited data on the role of LGE in primary MR.

Methods Patients with moderate or severe primary degenerative MR were prospectively recruited for multiparametric cardiac MRI (1.5 Tesla scanner Magnetom Avanto, Siemens) and cardiopulmonary exercise stress echocardiography (Epic, Phillips). All patients were asymptomatic without a Class 1 indication for surgery. Patients with coronary artery disease or significant aortic valve disease were excluded.

Results LGE was present in 18 (30\%) out of 61 patients (table 1). This was isolated to the point of insertion of the right ventricle (RV) into the septum in 8 cases. In the remaining 10 cases, LGE was located in the base of the left ventricle (LV; figure 1) or the papillary muscle heads. The presence of LGE was significantly associated with more severe MR, RV dilatation and reduced RV ejection fraction but no difference in LV size (LVEDV $92 \pm 22 \mathrm{ml} / \mathrm{m}^{2}$ vs $\left.99 \pm 23 \mathrm{ml} / \mathrm{m} 2\right)$, mass $(64 \pm 16 \mathrm{~g}$ / $\mathrm{m}^{2}$ vs $67 \pm 10 \mathrm{~g} / \mathrm{m}^{2}$ ) or function (LVEF $71 \pm 8 \%$ vs $69 \pm 6 \%$ ) was found. Functionally, this translated to the presence of ventilation-perfusion mismatch (VE/VCO2), a measure of exercise induced pulmonary hypertension. There was no difference in the maximum exercise capacity of patients according to presence of LGE.

Conclusion We demonstrate LGE of the LV myocardium to be present in $30 \%$ of patients with asymptomatic moderate-severe primary degenerative $\mathrm{MR}$, and represents an early marker of

Kaplan Meier Analysis

(Composite end point: admission with ACS or for revascularisation)

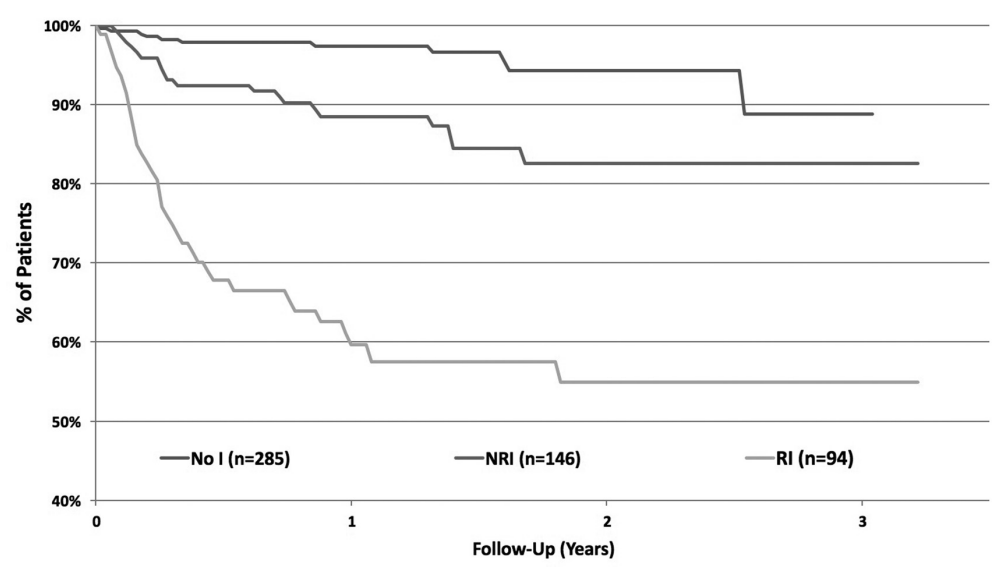

